\title{
MODELING EARTHQUAKE RISK VIA EXTREME VALUE THEORY AND PRICING THE RESPECTIVE CATASTROPHE BONDS*
}

\author{
BY
}

\author{
Alexandros A. Zimbidis, Nickolaos E. Frangos \\ and Athanasios A. Pantelous
}

\begin{abstract}
The aim of the paper is twofold. Firstly, to analyze the historical data of the earthquakes in the boarder area of Greece and then to produce a reliable model for the risk dynamics of the magnitude of the earthquakes, using advanced techniques from the Extreme Value Theory. Secondly, to discuss briefly the relevant theory of incomplete markets and price earthquake catastrophe bonds, combining the model found for the earthquake risk and an appropriate model for the interest rate dynamics in an incomplete market framework. The paper ends by providing some numerical results using Monte Carlo simulation techniques and stochastic iterative equations.
\end{abstract}

\section{KEYWORDS}

Extreme Value Theory, Catastrophe Bonds, Monte Carlo Simulation, Iterative Stochastic Equations, Incomplete Market.

\section{INTRODUCTION}

Catastrophe risks are related to extreme events which have low-probability (which means that they can not be easily predicted) but relatively huge negative economic consequences. These severe undesirable economic characteristics are normally found in natural disasters and have forced many insurers to find an appropriate way to limit the respective amount of losses and transfer the retained catastrophe risk.

In recent occurrences of the major catastrophic events in North America, insurers and reinsurers have had the sufficient capital to meet successfully their obligations, according to the Reinsurance Association of America (R.A.A., Nuter Fraklin, 2002). For instance, after hurricane Andew in 1992 with losses

\footnotetext{
* This work was supported by the reinforcement program of Human Research Manpower \#8211 "PENED" in the framework of Measure 8.3, Action 8.3.1 of the Operational program of competitiveness \#8211; Third Community Support Program.
} 
of $\$ 19.6$ billion, the Northridge earthquake in 1994 with $\$ 14.9$ billion (R.A.A., 2002), the hurricanes Chares, Frances, Ivan and Jeanne in 2004 with $\$ 20$ billion (United States General Accounting Office: G.A.O. 2005), the three largest natural disasters on record in the U.S.A. before the recently hurricanes Katrina and Rita in 2005 with $\$ 23$ billion (G.A.O. 2006) which hit the south east coasts, not one reinsurer went insolvent or failed to pay a claim as a result of an insolvency or financial distress. Moreover, the reinsurance industry succeeded to face the largest insured loss ever happened (as a result of a man-made catastrophe) of the September 11, 2001 terrorist attacks. For the last event, the insured losses amounted approximately to $\$ 60$ billion (R.A.A., 2002), with the reinsurance industry paying the $65 \%$ of that total.

Although traditional reinsurance providers have had the sufficient capital to meet their obligations out of the extreme catastrophic events, the insurance and reinsurance industry was constantly looking for alternative ways to spread the risk. In 1992, a new financial invention described as: catastrophe risk securitization, established with the introduction of a series of index-linked catastrophe loss futures and options by the Chicago Board of Trade (C.B.O.T). Those securities were a supplement to traditional reinsurance market mainly for: i) high layer coverage, for very rare events where they provide some experimental capacity on the fringe of the traditional market capacity and ii) where direct indemnity for losses is less important to the ceding companies. The relatively few securitizations actually put in effect in the last 5 years makes clear that insurers view securitization as a supplement to reinsurance, and not as a replacement (R.A.A., Nuter Fraklin, 2002).

Those option and future contracts were among the first attempts to market natural disaster-related securities, supporting the traditional reinsurance. They offered the advantage of standardization with low transaction costs traded over an exchange. Particularly, the purchasers of a catastrophe risk option pay to the sellers a premium, and the sellers provide the purchasers with cash payment if an index measuring insurance industry catastrophe losses exceeds a certain fixed level. So, while the catastrophe loss index remains below a specified level for the prescribed time period, the option expires worthless, and the sellers keep the premium. The option (and futures respectively) may have been purchased by an insurance company which requires hedging its catastrophe risk and may have been sold by firms which outperform in the event of a catastrophe - for example, construction companies - or by investors looking for a chance to diversify outside of traditional securities markets, extensive analysis provided by several papers, including D'Arcy \& France (1992), Cummins \& Geman (1995) and O'Brien (1997). Although, catastrophe option (and future) contracts were revised several times and covered exposures on national, regional and state base, because of the low trading which raised questions about liquidity, forced the C.B.O.T to cease them in 1999 and one year later, in 2000, to delist them (GAO, 2002).

Other risk-linked securities, which were created and used in the mid-1990s in the aftermath of the two largest natural disasters on record in the U.S.A., 
were Catastrophe Bonds (CAT bonds), which is also named as an "Act of God bond" or "insurance-linked bond". According to Swiss Re Capital Markets data, the value of outstanding CAT bonds increased substantially from 1997 through 2004 (figure 1) about 615\%. However, the value of $\$ 4.3$ billion was small compared to industry catastrophe exposures. For instance, a 5 Saffir-Simpson scale hurricane striking densely populated regions of Florida alone could cause more than an estimated $\$ 50$ billion in insured losses (GAO, 2005).

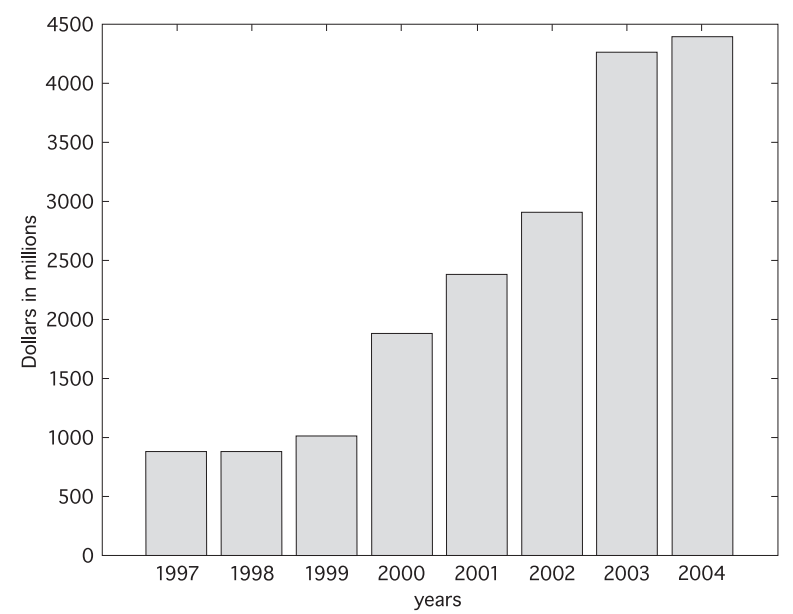

Figure 1: Catastrophe Bond Amount Outstanding, Year-end 1997-2004. (Source: GAO analysis of Swiss Re Capital Markets data).

Currently, most risk-linked securities are CAT bonds which contain brunches that have received particular investment grade ratings. One characteristic example of this kind is Swiss Re Mortality bond. Swiss Re set up a special bond, dubbed Vita Capital Ltd., which issued $\$ 400$ million in mortality-indexed notes. The proceeds were placed in a collateral account and invested in high-quality securities, which returns were swapped into a Libor-based return. The notes are expected to mature on January 1, 2007, at par. However, if mortality in five countries increases to pre-defined levels, investors of the notes may receive less than par or even zero at maturity. Note holders will receive quarterly interest payments of Libor plus 1.35\% (annualized) in return for bearing extreme mortality risk, see Beelders O. \& Colarossi D. (2004). Figure 1 contains a graphical description of the issuance structure.

Under the terms of non indemnity-based catastrophe bonds, for the sponsoring insurance company to collect part or the investors' entire principal when the catastrophe occurs, an independent third party must confirm that the objective catastrophic event was met, such as an earthquake reaching i.e. 7.0 in local magnitude as reported by the National Geological Survey. Moreover, the Bond Market Association (B.M.A, 2002) commented that there are often compelling reasons for sponsors of the risk-linked securities to use non indemnity-based 


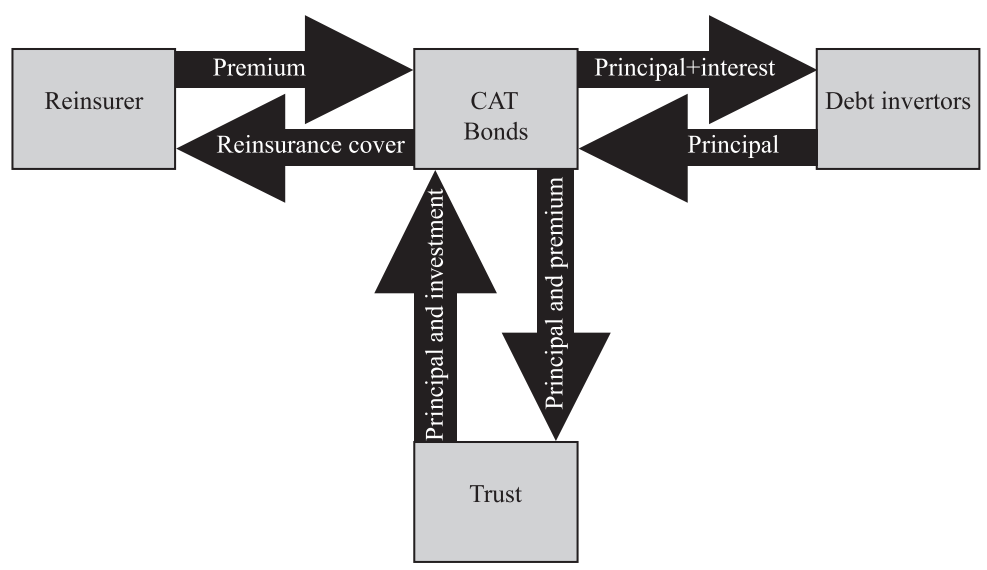

Figure 2: The structure of the CAT bonds (Source: GAO).

structures. For instance, they can more effectively shield the confidentiality of the sponsor's underwriting criteria and provide for more streamlined deal structuring and deal execution, as well. Moreover they may facilitate a more rapid payout in response to triggering events.

In this paper we will use the special framework explained above to create a CAT bond for the earthquakes placed in the Greek boarder area. Thus, the creation of a CAT bond for Greece provides a secure mechanism for direct transfer of major catastrophic earthquakes' casualties to capital markets. This is one way to debilitate the homeowners' insurance market and keep earthquake insurance available at affordable prices.

A brief outline of the paper follows. Section 2 provides a quick overview of the theory for pricing catastrophe bonds, for incomplete markets and builds two models: one-period and multi-period, for pricing process of CAT bonds. Section 3 relies extensively on Extreme Value Theory. We calculate the distribution function of the annual maximum magnitude of earthquakes in the boarder area of Greece for the period 1966-2005. In section 4, we design a special 5-year period CAT bond and obtain (using Monte Carlo simulation techniques and Iterative Stochastic Equations) the appropriate price. The final section 5 provides a general overview of the results and reveals other potential directions for further research.

\section{Modeling CAtastrophe Bonds}

\subsection{Pricing Catastrophe Bonds and Incomplete Markets}

There are several important articles that focus on the pricing of catastrophelinked securities. For instance, in the research work of Briys (1997) a simple formula for non-indemnity insurance-linked bonds is derived in an arbitrage-free 
framework. Loubergé, Kellezi \& Gilli (1999) provide a numerical estimation of the pricing of CAT bonds under the assumptions that the catastrophe loss follows a pure Poisson process, the loss severity is an independently identical lognormal distribution, and the interest rate is driven by a binomial random process. In the same way, Baryshnikov, Mayo and Taylor (2001) present an arbitrage-free solution to the pricing of CAT bonds under the assumption of (almost) continuous trading. They use the compound Poisson for underlying the CAT bond and the pure Poisson point process both for the occurrence and the economic effect of catastrophes. Lee and Yu (2002) develop a contingent claim model to price a default-risky, catastrophe-linked bond. However, they are primarily interested in analyzing default risk, and therefore, to specialize their results to the case where CAT bonds are directly issued by insurers. Recently, Vaugirard $(2003,2004)$ vindicate the existence of well-defined arbitrage prices for CAT bonds, not withstanding a framework of incomplete markets and non-traded underlying state variables. It is showed that their valuation comes down to computing first-passage time distributions, since bondholders are showed to be in a short position on one-touch digital options based on risktracking indices that follow jump-diffusion processes in a Gaussian interest rate framework.

Although, the general problem of pricing a security may be well manipulated within the framework of a complete market using the no-arbitrage theory, the payoffs from insurance-based securities, whose cash flows may depend on earthquakes or other catastrophic events, can not be closely approximated by an appropriate portfolio of the traditional assets that already traded in the market such as stocks and corporate bonds, Cox \& Pedersen (2000). Consequently, the pricing of a CAT bond requires an incomplete market framework.

In the case of incomplete markets, there is no "universal" theory to date that successfully addresses all aspects of pricing, such as specification of hedging strategies and robustness of prices, Young V. (2004). For that reason, various alternative pricing mechanisms have been developed that are tied to the specific nature of each market.

Fortunately, the fact that catastrophe risk is uncorrelated with movements in underlying economic variables renders the incomplete market theory somewhat simpler than the case of significant correlation, Cox \& Pedersen (2000). We use this approach and the theory of equilibrium pricing to develop a simple one-period and one more complicated multi-period model for pricing catastrophe bonds.

Actually, the valuation is performed in two stages. The first stage with respect to the estimation of risk dynamics is presented in section 3, i.e. the distribution function of the annual maximum earthquakes of the boarder area of Greece. The statistical analysis of extremes is a key factor to many of the risk management problems related to Insurance, Reinsurance and generally speaking in Finance. In this paper we develop a model using the tools of Extreme Value Theory. The second stage requires the selection or estimation of the interest rate dynamics. 


\subsection{One period (basic) Model}

In this subsection we proceed with the construction of the simple one-period model where the interest rate dynamics are restricted to constant values of different rates.

Firstly, we define the necessary symbols and the respective notation keeping in mind the discrete framework of our analysis, i.e.

$K:$ is the face amount of the CAT bond.

$r$ : is the risk free rate continuously compounding (up to maturity date).

$e:$ is the extra premium loading for bearing earthquake risk (Normally, this is a positive quantity reflecting the respective risk aversion of the buyers of such a security)

$R:$ is the basic element for the determination of the coupon payment rate for the one year period as long as a specified catastrophic event does not occur.

$M:$ is the maximum magnitude level of the earthquake in the boarder area of Greece. $M$ is a random variable following the distribution obtained in Section 3 (see Table 2). Moreover, $M$ is measured in local magnitude (LM) scale.

$P:$ is the price of the CAT bond

$C:$ is the cash value of the CAT bond at the maturity date depending upon the value of $M$ according to the structure described in the following expression (2.2.1).

$$
C= \begin{cases}K \cdot(1+3 R), & \text { if } M \in[0,5.4] \\ K \cdot(1+2 R), & \text { if } M \in[5.4,5.8] \\ K \cdot(1+R), & \text { if } M \in[5.8,6.2] \\ K, & \text { if } M \in[6.2,6.6] \\ \frac{2}{3} K, & \text { if } M \in[6.6,7.0] \\ \frac{1}{3} K, & \text { if } M \in[7.0,7.4] \\ 0, & \text { if } M \in[7.4, \infty]\end{cases}
$$

Assuming that $K, r, R$ and $e$ are constants. And according to standard equivalence principle the price of the CAT bond is obtained as

$$
P=E_{Q_{1}}\left(e^{-(r+e)} \cdot C\right)
$$

where $Q_{1}$ is the probability measure corresponding to the distribution for $M$ obtained in next section (see Table 2). A numerical example is provided in section 4. 


\begin{tabular}{cc}
$\mathrm{P}$ & $\mathrm{C}$ \\
\hline & 1 \\
Issue Date & Maturity Date \\
FIGURE 3: The diagram for the one period model.
\end{tabular}

\subsection{Multi period (advanced) Model}

Now, we proceed with the formulation of the multi period model defining all the necessary symbols and the respective notation, still keeping in mind the discrete framework of our analysis, i.e.

$K, r$ and $e$ have the same meaning as in the one period model

$T$ : is the maturity date of the CAT bond

$i_{n}$ : is the annual rate of a typical bank deposit account return for the $(n+1)$-th year (i.e. in the interval $[n, n+1)$ ). We assume that $i_{n}$ is a log-normally distributed random variable (that assumption coincides with the practical experience, see for further details: Kellison, 1991 and Baxter \& Rennie, 1999) with parameters $\mu_{1}$ and $\sigma_{1}^{2}$ for any $n=1,2,3, \ldots$

$$
E\left(i_{n}\right)=e^{\mu_{1}+\frac{1}{2} \sigma_{1}^{2}}=2 \% \quad \& \quad \operatorname{Var}\left(i_{n}\right)=e^{2 \mu_{1}+\sigma_{1}^{2}}\left(e^{\sigma_{1}^{2}}-1\right)=(0.5 \%)^{2}
$$

Thus,

$$
i_{n}: \operatorname{LogNormal}(-3.94,0.061)
$$

$R_{t}$ : is the Euribor rate for 12 months lending at time $t$. Due to popularity we assume that $\left\{R_{t}, t \in[0, T]\right\}$ is a stochastic process driven by a Geometrical Brownian motion, see Baxter \& Rennie (1999), Øksendal (2003), Romaniuk (2003) and others, described by the following differential equation:

$$
d R_{t}=\mu_{2} R_{t} d t+\sigma_{2} R_{t} d W_{t}
$$

where $\mu_{2}, \sigma_{2}>0$, are constants corresponding to the drift and the volatility parameters and $W_{t}$ is the standard arithmetic Brownian motion according to a physical measure $Q_{2}$. So,

$$
R_{t}=R_{o} \exp \left(\left(\mu_{2}-\frac{1}{2} \sigma_{2}^{2}\right) t+\sigma_{2} W_{t}\right)
$$

$R^{(j)}$ : is the $\mathrm{j}$-th path (realization) of the Euribor rate $\left\{R_{t}, t \in[0, T]\right\}$ process.

$M_{n}$ : is the annual maximum magnitude level of the earthquake in the boarder area of Greece in the $\mathrm{n}$-th year. The $M_{n}$ 's are measured in local magnitude (LM) scale and have the common distribution described in Section 3 (see Table 2). 
$M^{(j)}$ : is the j-th path (realization) of the $\left\{M_{n}, n=1,2, \ldots\right\}$ process for the annual maximum magnitude of the earthquake.

$P:$ is the price of the CAT bond based on earthquakes, at time 0 .

$f\left(R_{l}, M_{l}\right)$ : is the cash value received by bondholder of the CAT bond at time $l=1,2, \ldots, T$ (see Figure 4).

$$
\begin{aligned}
& f\left(R_{l}, M_{l}\right)=\left\{\begin{array}{l}
\left(3 R_{l}\right) \cdot \mathbf{1}_{\left[0<M_{1} \leq 5.4\right]}+\left(2 R_{l}\right) \cdot \mathbf{1}_{\left[5.4<M_{1} \leq 5.8\right]}+\left(R_{l}\right) \cdot \mathbf{1}_{\left[5.8<M_{1} \leq 6.2\right]} \\
l=1,2,3, \ldots, T-1 \\
\left(3 R_{l}\right) \cdot \mathbf{1}_{\left[0<M_{1} \leq 5.4\right]}+\left(2 R_{l}\right) \cdot \mathbf{1}_{\left[5.4<M_{1} \leq 5.8\right]}+\left(R_{l}\right) \cdot \mathbf{1}_{\left[5.8<M_{1} \leq 6.2\right]}+ \\
+K \cdot \mathbf{1}_{\left[5<\max \left(M_{1}\right) \leq 6.6\right]}+\frac{2}{3} K \cdot \mathbf{1}_{\left[6.6<\max \left(M_{1}\right) \leq 7.0\right]}+\frac{1}{3} K \cdot \mathbf{1}_{\left[7.0<\max \left(M_{1}\right) \leq 7.4\right]} \\
l=T
\end{array}\right. \\
& \begin{array}{cccccccc}
\mathrm{P} & f\left(R_{1}, M_{1}\right) & f\left(R_{2}, M_{2}\right) & f\left(R_{l}, M_{l}\right) & & f\left(R_{T-1}, M_{T-1}\right) & f\left(R_{T}, M_{T}\right) \\
\hline & \mid & \mid & & \mid & & \mid & \mid \\
0 & 1 & 2 & \ldots & 1 & \ldots & T-1 & T
\end{array}
\end{aligned}
$$

FIGURE 4: The diagram for the cash-flow stream of the multi-period model.

It is obvious that a catastrophe might or might not occur prior to the scheduled maturity date, at time $T$. As we can see from the cash-flow stream to the bondholders, in expression (2.3.6), the CAT bond with the face amount of $€ K$ is scheduled to make coupons payments of $€ K \cdot(3 R), € K \cdot(2 R), € K \cdot R$ at the end of each period if magnitude level of earthquake is between $(0,5.4]$, $(5.4,5.8],(5.8,6.2]$, respectively or zero coupon payment if the magnitude of the earthquake exceeds the level of 6.2. While the CAT bond is scheduled to repay all, the two thirds, a third or nothing from the capital at maturity day if the maximum magnitude level of earthquakes during that period, let us say $T$-year time period, is between $(0,6.6],(6.6,7.0],(7.0,7.4]$ and $(7.4, \infty]$, respectively.

Before we go further, we present some technical details in order to obtain a concrete theoretical basis for our problem.

We model the financial market and consequently the process for the Euribor rate $R_{t}, t \in(0, T)$ within a complete probability space $\left(\Omega_{2}, F_{2}, Q_{2}\right)$ which is equipped with the appropriate natural filtration.

The catastrophic risk is modeled via a discrete process $\left\{m_{n}, n=1,2, \ldots\right\}$ for the annual maximum magnitude level of the earthquake in the boarder area of Greece within another complete probability space $\left(\Omega_{1}, F_{1}, Q_{1}\right)$ also equipped with the appropriate natural filtration. 
Hence, the probability space for the full model is the triple $(\Omega, F, Q)$, where:

- the sample space for our model is the following product: $\Omega=\Omega_{1} \times \Omega_{2}$. Therefore, a typical element of the sample space for the full model is of the form:

$$
\omega=\left(\omega_{1}, \omega_{2}\right) \text { with } \omega_{1} \in \Omega_{1}, \omega_{2} \in \Omega_{2}
$$

- the filtration for the product measure space is denoted by the rectangular:

$$
F=F_{1} \times F_{2}
$$

- and the probability measure on the sample space $\Omega$ is given by the natural product measure structure. Therefore, the probability of a generic state of the world, $\omega=\left(\omega_{1}, \omega_{2}\right)$, is

$$
Q(\omega)=Q_{1}\left(\omega_{1}\right) \cdot Q_{2}\left(\omega_{2}\right)
$$

This assumption implies the independence among the events depending only on economic risk variables and those depending only on catastrophe risk variables. Hence, the time occurrence of the catastrophe is independent of the term structure.

Now, according to equilibrium pricing theory we obtain the price $P$ as

$$
P:=E_{Q}\left\{e^{-(r+e) T} \sum_{l=1}^{T} F V_{T} f\left(R_{l}, M_{l}\right)\right\}
$$

where

$$
F V_{T} f\left(R_{l}, M_{l}\right)=f\left(R_{l}, M_{l}\right) \cdot \prod_{k=0}^{T-l-1}\left(i+i_{l+k}\right)
$$

Assuming that expectation (2.3.5) exists, we can approximate the price of the CAT bond as

$$
P=\lim _{m \rightarrow \infty} P^{(m)}
$$

where

$$
P^{(m)}=e^{-(r+e) T} \frac{1}{m} \sum_{j=1}^{m} \sum_{l=1}^{T} F V_{T}\left(R_{l}^{(j)}, M_{i}^{(j)}\right)
$$

Therefore, the real price $P$ at issue time may be calculated using Monte Carlo methods, Boyle, Broadie \& Glassermann (1997), Romaniuk (2003) and this project is addressed to the section 4 . 


\section{Discussion of the Greek Earthquake Data}

\subsection{Extreme Value Theory and Modeling}

The model focuses on the statistical behavior of maxima.

$$
M_{n}=\max \left\{X_{1 n}, X_{2 n}, \ldots, X_{m n}\right\}
$$

where $X_{1 n}, X_{2 n}, \ldots, X_{m n}$, is the sequence of $m=365$ independent random variables having a common unknown distribution function (d.f.) $F$ and measures the magnitude of earthquakes during the 365 days of each year in the boarder area of Greece for the period $[n, n+1)$. So the sequence of $M_{n}$ represents the $n^{\text {th }}$ annual maximum of the process over 40 years of observation (see Table 1).

In theory, the distribution of $M_{n}$ can be derived exactly for all values of $n$ :

$$
\begin{aligned}
\operatorname{Pr}\left[M_{n} \leq z\right] & =\operatorname{Pr}\left[X_{1 n} \leq z, \ldots, X_{m n} \leq z\right] \\
& \stackrel{\text { iid } X_{i}}{=} \operatorname{Pr}\left[X_{1 n} \leq z\right] \cdot K \cdot \operatorname{Pr}\left[X_{m n} \leq z\right]=\{F(z)\}^{m}
\end{aligned}
$$

However, this is not immediately helpful in practice, since the distribution function $F$ is still unknown. There have been developed two well known statistical methods to overcome this problem. The first standard technique estimates $F$ from the observed data, and then substitute this estimate into (3.1.2). As an alternative approach, we accept that $F$ is unknown and search for approximate families of models for $F^{m}$, which can be estimated on the basis of the extreme data only. This is quite similar in practice with the approximation of the distribution of sample mean by the Normal distribution, as justified by the Central Limit Theorem (C.L.T.). So, the arguments are essentially an extreme value ana$\log$ of the C.L.T.

The entire range of possible limit distributions for the rescaled sample maxima $M_{n}^{*}=\frac{M_{n}-b_{n}}{a_{n}}$ is provided by the well known following Theorem of Fisher Tippett, Gnedenko (Fisher \& Tippett, 1928; Gnedenko, 1943; Embrechts, Klüppelberg \& Mikosch, 2003: Theorem 3.2.3 and Coles, 2004: Theorem 3.1).

Theorem 3.1.1 (Fisher - Tippett, Gnedenko)

If exist sequences of constants $\left\{a_{n}: a_{n}>0 \forall n \in \mathbb{N}\right\}$ and $\left\{b_{n}\right\}_{n \in ¥}$ such that:

$$
\operatorname{Pr}\left[M_{n}^{*} \leq z\right]=\operatorname{Pr}\left[\frac{M_{n}-b_{n}}{a_{n}} \leq z\right] \rightarrow G(z) \text { as } n \rightarrow \infty, z \in \mathbb{R}
$$

(where $G$ is a non-degenerate distribution function) then $G$ belongs to one of the following families.

$$
\text { I. (Gumbel) } G(z)=\exp \left\{-\exp \left[-\left(\frac{z-b}{a}\right)\right]\right\}, \quad-\infty<z<\infty
$$


II. (Fréchet) $\quad G(z)= \begin{cases}0, & z \leq b \\ \exp \left\{-\left[-\left(\frac{z-b}{a}\right)\right]^{-\gamma}\right\}, & z>b\end{cases}$
III. (Weibull) $\quad G(z)= \begin{cases}\exp \left\{-\left[-\left(\frac{z-b}{a}\right)\right]^{\gamma}\right\}, & z<b \\ 1, & z \leq b\end{cases}$ for parameters $a>0, b$ and in the case of families II and III, $\gamma>0$.

The unification of the previous three families of extreme value distribution into a single family simplified a lot the statistical implementation. Through the inference of a new parameter $\xi$, the data determine by themselves the most appropriate type of tail behavior, so we avoid completely making a priori judgment for the individual extreme value family. Moreover, uncertainty in the inferred value of $\xi$ succeeds in measuring the lack of certainty as to which of the original three types is most appropriate for a given dataset. For convenience we restate Theorem 3.1.1 in the following modified form.

Theorem 3.1.2 (Fisher - Tippett, Gnedenko)

If exist sequences of constants $\left\{a_{n}: a_{n}>0 \forall n \in \mathbb{N}\right\}$ and $\left\{b_{n}\right\}_{n \in ¥}$ such that:

$$
\operatorname{Pr}\left[M_{n}^{*} \leq z\right]=\operatorname{Pr}\left[\frac{M_{n}-b_{n}}{a_{n}} \leq z\right] \rightarrow G(z) \text { as } n \rightarrow \infty, z \in \mathbb{R}
$$

for a non-degenerate distribution function $G$, then $G$ is a member of the Generalized Extreme Value (GEV) family of distributions or von Mises type Extreme Value distribution or the von Mises-Jenkinson type distribution.

$$
G(z)=\exp \left\{-\left[1+\xi\left(\frac{z-\mu}{\sigma}\right)\right]^{-\frac{1}{\xi}}\right\}
$$

defined on the set $\{z: 1+\xi(z-\mu) / \sigma>0\}$, where the parameters satisfy $-\infty<$ $\mu<\infty, \sigma>0$, and $-\infty<\xi<\infty$.

The model has three parameters: $\xi$ is a shape parameter, $\mu$ is a location parameter and $\sigma$ is scale parameter. The type II and type III classes of extreme value distribution correspond respectively to the cases $\xi>0$ and $\xi<0$ in the parameterization. The subset of the GEV family with $\xi=0$ is interpreted as the limit of (3.1.3) as $\xi \rightarrow 0$, leading to the Gumbel family.

We complete this subsection by explaining the estimation technique for the parameters $(\xi, \sigma, \mu)$. There are many considerable techniques, as the graphical techniques which are based on versions of probability plots, the moment-based 
techniques in which the parameters are estimated as specified functions of order statistics and the classical likelihood based method. Each technique has its particular characteristics, but the undisputable value and adaptability to more complex model-building of the likelihood method makes it particular attractive.

The maximum likelihood estimation for the parameters $(\xi, \sigma, \mu)$ has been studied by a number of authors including Jenkinson (1969), Prescott and Walden (1980, 1983), Hosking (1985) and Macleod (1989). Moreover, it should be mentioned that the regularity conditions are satisfied when $\xi>-1 / 2$ and in this case the asymptotic variances and covariances of the maximum likelihood estimators are given by the elements of the Fisher inverse information matrix, for further details see Kotz \& Nadarajah (2002), Coles (2004). Fortunately, the experience with real-world data, as the data from the annual maximum earthquakes of Greece analyzed in the next subsection, suggests the above condition for $\xi$ is almost always satisfied.

So, we assume that $M_{1}, \ldots, M_{n}$ are independent variables having the GEV distribution. Then, the log-likelihood for the GEV parameters is given by the following expression (3.1.4) when $\xi \neq 0$.

$$
\begin{aligned}
l(\xi, \sigma, \mu)= & n \log \sigma-(1+1 / \xi) \sum_{i=1}^{n} \log \left[1+\xi\left(\frac{M_{i}-\mu}{\sigma}\right)\right] \\
& -\sum_{i=1}^{n}\left[1+\xi\left(\frac{M_{i}-\mu}{\sigma}\right)\right]^{-1 / \xi}
\end{aligned}
$$

provided that

$$
1+\xi\left(\frac{M_{i}-\mu}{\sigma}\right)>0, \text { for } i=1,2, \ldots, n
$$

The expression (3.1.5) is very important because it provides a compact relationship for the three parameters. At least when one of the observed data falls beyond an end-point of the distribution, the likelihood is zero and consequently the log-likelihood equals to $-\infty$.

The maximization of equation (3.1.4) with respect to the parameter vector $(\xi, \sigma, \mu)$ leads to the maximum likelihood estimate with respect to the entire GEV family. There is no analytic solution, but for any given dataset the maximization is obtained straightforward by using standard numerical algorithms (e.g. Newton-Raphson method), Coles (2004). From the programming point of view, Hosking (1985) has provided a FORTRAN subroutine MLEGEV that facilitates the calculation of the maximum likelihood estimates of the parameter vector $(\xi, \sigma, \mu)$ and the variance-covariance matrix of the estimated parameters. McNeil $(1997,2001)$ has created a library of S-PLUS functions for implementing Extreme Value Theory (EVT) including subroutines for GEV distributions with the code name EVIS, Version 4. Although, in the next subsection 3.2 we use Coles (2004) GEV.FIT function for finding maximum likelihood estimators of the GEV model. 


\subsection{Data Analysis}

This analysis is based on the series of annual maximum magnitude of the earthquakes in the boarder area of Greece, over the period 1966-2005 as described in the following Table 1, and the presentation of the elements based on Coles (2004). From Figure 5, it seems reasonable to assume that the pattern of variation has stayed constant over the observation period, so we model the data as independent observations from the GEV distribution.

TABLE 1

Annual Maximum Earthquakes in the Boarder Area of Greece

\begin{tabular}{ll|ll|ll|ll}
\hline \hline 1966 & 5.1 & 1976 & 5.7 & 1986 & 5.5 & 1996 & 5.9 \\
1967 & 6.3 & 1977 & 6.1 & 1987 & 5.4 & 1997 & 6.1 \\
1968 & 6.7 & 1978 & 6.1 & 1988 & 5.5 & 1998 & 5.5 \\
1969 & 6.3 & 1979 & 5.5 & 1989 & 5.4 & 1999 & 5.9 \\
1970 & 5.4 & 1980 & 6.3 & 1990 & 5.5 & 2000 & 6.4 \\
1971 & 5.1 & 1981 & 6.3 & 1991 & 5.3 & 2001 & 5.3 \\
1972 & 6.0 & 1982 & 6.3 & 1992 & 5.8 & 2002 & 6.1 \\
1973 & 5.5 & 1983 & 6.6 & 1993 & 5.4 & 2003 & 5.9 \\
1974 & 5.5 & 1984 & 5.9 & 1994 & 5.9 & 2004 & 6.0 \\
1975 & 5.6 & 1985 & 5.3 & 1995 & 6.1 & 2005 & 5.7 \\
\hline \hline
\end{tabular}

A Revised Catalogue of Earthquakes in the Boarder Area of Greece for the Period 1966-2000 \& Site Data from the Institute of Geodynamics, National Observatory of Athens (IG-NOA) for the Period 2001-2005.

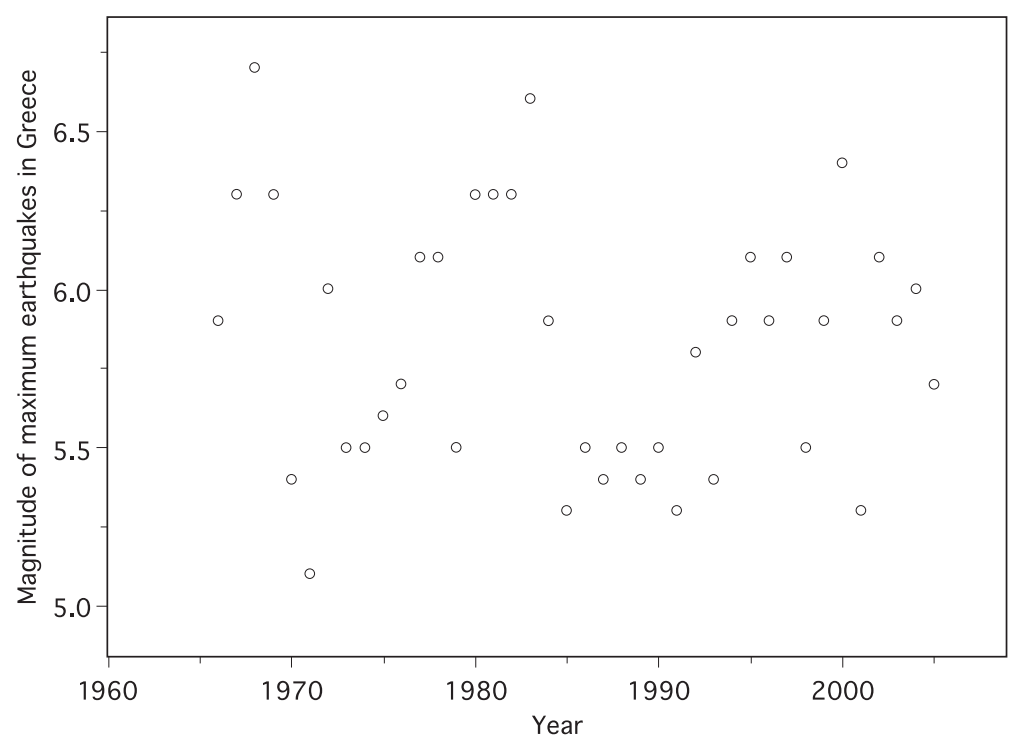

FIgURE 5: Scatter Plot of the Annual maximum magnitude Earthquakes of Greece. 
Maximization of the GEV log-likelihood for these data leads to the following estimate:

$$
(\hat{\xi}, \hat{\sigma}, \hat{\mu})=(-0.1977803,0.3656859,5.6708431)
$$

for which the log-likelihood is 18.75536 . The approximate variance-covariance matrix of the parameter estimates is

$$
V=\left(\begin{array}{ccc}
0.022310976 & -0.0045936353 & -0.0047245186 \\
-0.004593635 & 0.0025385622 & 0.0008426891 \\
-0.004724519 & 0.0008426891 & 0.0045365846
\end{array}\right)
$$

The diagonals of the variance-covariance matrix correspond to the variances of the individual parameters of $(\xi, \sigma, \mu)$. Computing square roots, the standard errors are $0.1493686,0.05038415$ and 0.06735417 for $\xi, \sigma$ and $\mu$ respectively. Combining estimates and standard errors, approximate $95 \%$ confidence intervals for each parameter are $[-0.50,0.10]$ for $\xi,[0.07,0.27]$ for $\sigma$ and $[5.57,5.77]$ for $\mu$. In particular, although the maximum log-likelihood estimate for $\xi$ is negative, corresponding to a bounded distribution, the $95 \%$ confidence interval extends well above zero, so that the strength of evidence from the data for a bounded distribution is not strong. Greater accuracy of confidence intervals can usually be achieved by the use of profile likelihood. Figure 6 shows the profile log-likelihood for $\xi$, from which a $95 \%$ confidence interval is obtained, which is only slightly different to the earlier calculation.

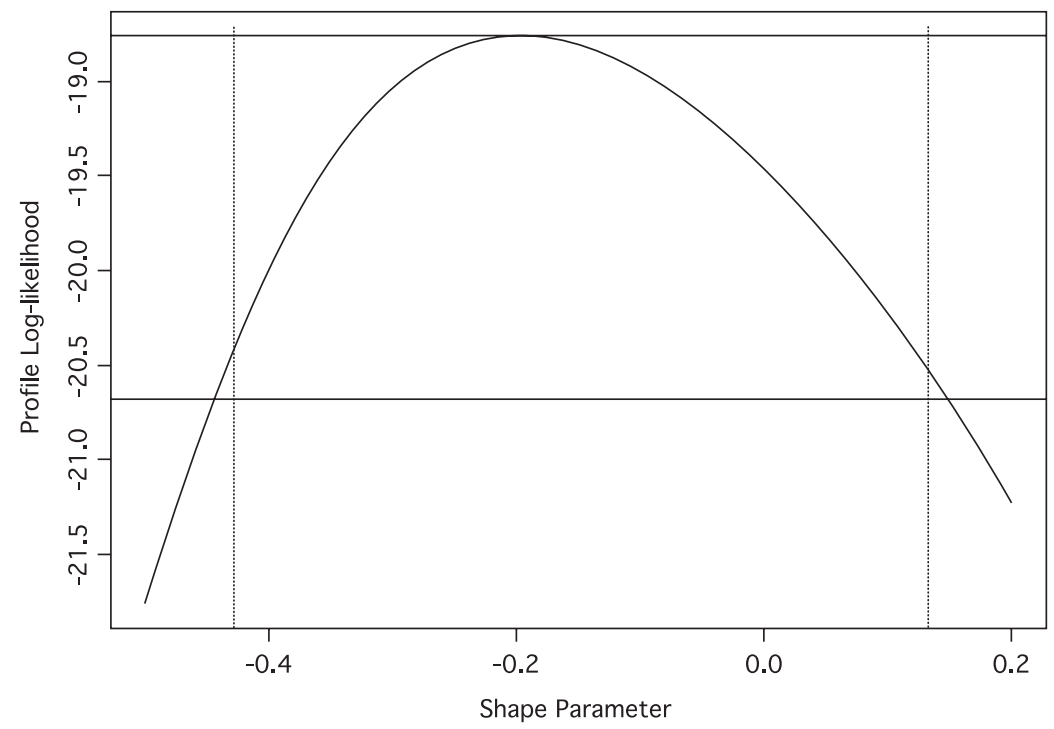

FIGURE 6: Profile log-likelihood for $\xi$ for the Annual Maximum Earthquakes of Greece. 
The various diagnostic plots for assessing the accuracy of the GEV model fitted to the Annual Maximum Earthquakes of Greece data are shown in Figure 7. Neither the probability plot nor the quantile plot give cause to doubt the validity of the fitted model: each set of plotted points is near-linear. The return level curve converges asymptotically to a finite level as a consequence of the negative estimate of $\xi$, though the estimate is close to zero and the respective estimated curve is close to a straight line. The curve also provides a satisfactory representation of the empirical estimates, especially once sampling variability is taken into account. Finally, the corresponding density estimate seems consistent with the histogram of the data. Consequently, all four diagnostic plots provide support to the fitted GEV model.

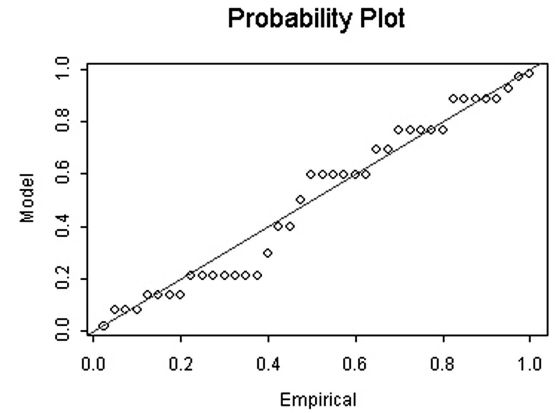

Return Level Plot

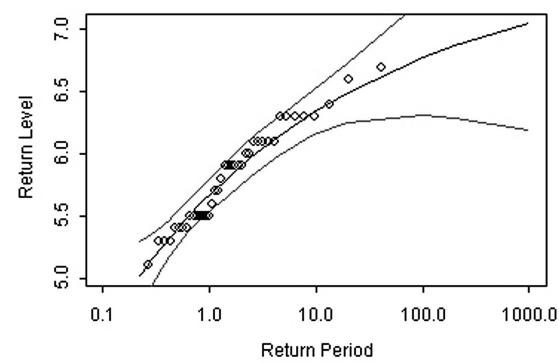

Quantile Plot

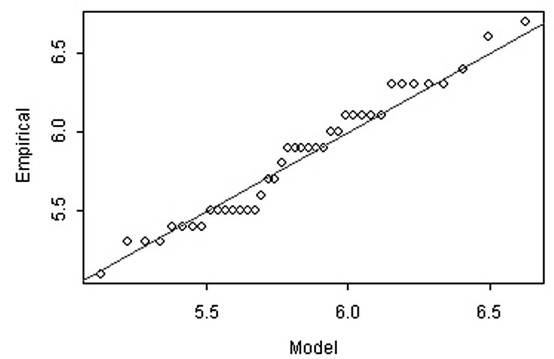

Density Plot

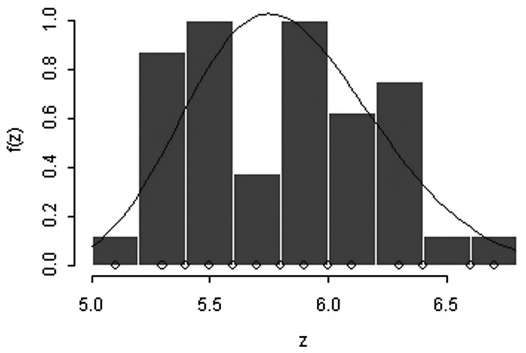

FIGURE 7: Diagnostic plots for GEV fit to the Annual Maximum Earthquakes of Greece.

Moreover, a further graphical tool which is the plot of the sample mean excess function, see Figure 8, provides an important sign of the tail behavior of the distribution, as it is explained in Beirlant, Teugels \& Vynckier (1996), Embrechts, Klüppelberg \& Mikosch (2003) and others. It is a plot of this kind: $\left\{\left(u, \hat{e}_{n}(u)\right.\right.$, $\left.\left.M_{(1)}<u<M_{(n)}\right)\right\}$, where $M_{(1)}$ and $M_{(n)}$ are the first and the n-th order statistics and $\hat{e}_{n}(u)$ is the sample mean excess function defined as:

$$
\hat{e}_{n}(u)=\frac{\sum_{i=1}^{n}\left(X_{i}-u\right)^{+}}{\sum_{i=1}^{n} I_{\left\{X_{i}>u\right\}}}
$$


The sample mean excess function $\hat{e}_{n}(u)$ is an empirical estimate of the mean excess function which is defined as $e_{n}(u)=E[X-u \mid X>u]$. The mean excess function describes the estimated overshoot of a threshold given the exceedance occurs. If the points show an upward trend, then this is a clear sign of heavier tail behavior. Exponentially distributed data would give an approximately horizontal line and data from a short tailed distribution would show a downward trend. In particularly, as we can observe from Figure 6 the downward trend gives a strong evidence for a very short tail behavior for the Annual Maximum Earthquakes of the boarder area of Greece.

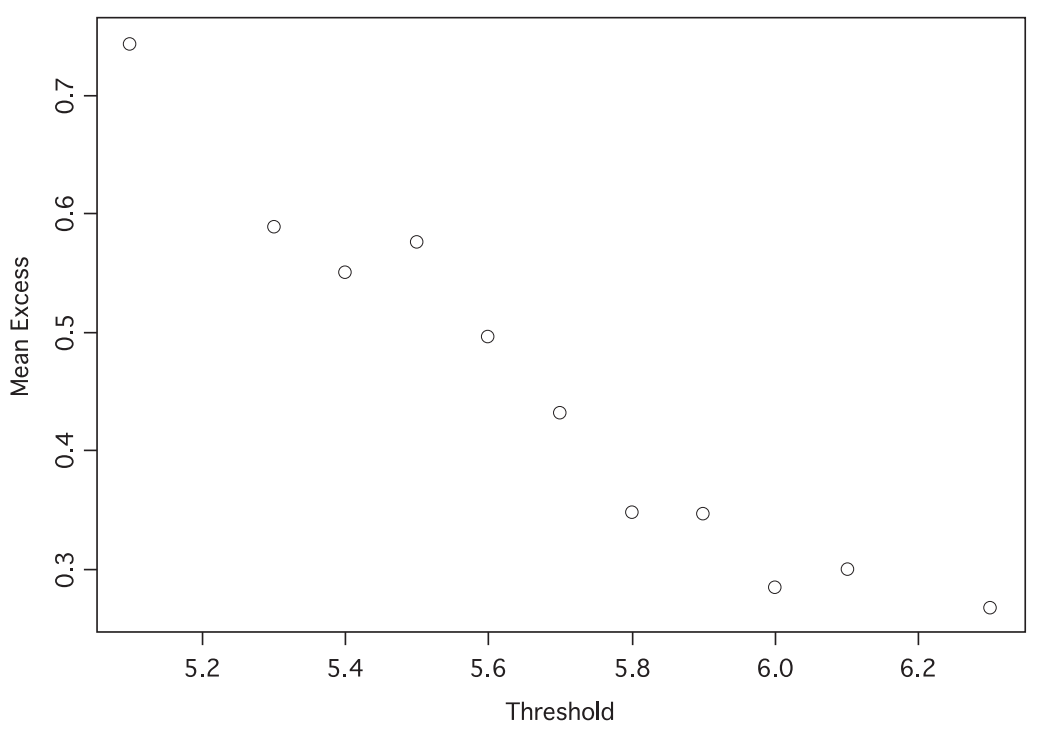

Figure 8: A sample mean excess plot of the Annual Maximum Earthquakes of Greece.

Finally, we determine the type of the limiting distribution for Annual Maximum Earthquakes of Greece, which is a type of Weibull (more precisely $G$ is called a standard extreme value distribution and have no similarity with the standard distribution Weibull whose extremal behavior is completely different, see more details in Embrechts, Klüppelberg \& Mikosch, 2003) and it is concentrated on $(-\infty, 0)$ : This type has the following form

$$
G(z)=\exp \left\{-\left[1+\xi\left(\frac{z-\mu}{\sigma}\right)\right]^{-\frac{1}{\xi}}\right\}
$$

where parameters are $(\hat{\xi}, \hat{\sigma}, \hat{\mu})=(-0.1977803,0.3656859,5.6708431)$.

Moreover, we notice that the extreme value distribution function is continuous on $\mathbb{R}$, hence $c_{n}^{-1}\left(M_{n}-d_{n}\right) \stackrel{d}{\rightarrow} G$ is equivalent to 


$$
\lim _{n \rightarrow \infty} P\left(M_{n} \leq c_{n} z+d_{n}\right)=\lim _{n \rightarrow \infty}\left\{F\left(c_{n} z+d_{n}\right)\right\}^{n}=G(z), \quad z \in \mathbb{R}
$$

and according to the convergence of type theorem (Theorem A1.5 of Embrechts, Klüppelberg \& Mikosch, 2003; see their book for further details) it is ensured that the limit law is uniquely determined up to any affine transformation.

In Table 2, we provide the exceedance probabilities intervals for the standard extreme value distribution (3.2.4)

TABLE 2

Exceedance Probabilities for the Model

\begin{tabular}{c|c|c|c|c|c|c}
\hline \hline $\mathrm{P}(5.0<\mathrm{M}<5.4)$ & $\mathrm{P}(5.4<\mathrm{M}<5.8)$ & $\mathrm{P}(5.8<\mathrm{M}<6.2)$ & $\mathrm{P}(6.2<\mathrm{M}<6.6)$ & $\mathrm{P}(6.6<\mathrm{M}<7.0)$ & $\mathrm{P}(7.0<\mathrm{M}<7.4)$ & $\mathrm{P}(\mathrm{M}>7.4)$ \\
0.1358699 & 0.3639984 & 0.3338672 & 0.1373934 & 0.0272371 & 0.001633007 & $9.7910^{-7}$ \\
\hline \hline
\end{tabular}

\section{Numerical Example And Simulation}

\subsection{Numerical Example for the one-period model}

Assuming the one period model and defining the variables $K=1,000 €, r=2.10 \%$, as for the one year Greek government bond (Bank of Greece, June 2005), $R=$ $2.08 \%$ and $e=5 \%$, we obtain according to expression (2.3.2) the price $P=952.5 €$.

\subsection{Simulation and Pricing for the multi-period model}

As a practical case study we describe a 5-year period CAT bond with payments depending on the magnitude of earthquakes in the boarder area of Greece as it has been described extensively in sections 2 and 3 .

We build our simulation using the following five steps.

\section{STEP 1}

Firstly, we generate 50,000 sequences of five arithmetic values from the GEV distribution representing the maximum magnitude of the earthquake for the 5 -year period up to the maturity date. This is relatively easily to do because we can invert the cumulative distribution function:

$$
G(z)=\exp \left\{-\left[1+\xi\left(\frac{z-\mu}{\sigma}\right)\right]^{-\frac{1}{\xi}}\right\}
$$

with parameters $(\hat{\xi}, \hat{\sigma}, \hat{\mu})=(-0.1977803,0.3656859,5.6708431)$.

Thus, we receive the expression:

$$
z=\mu+\left[\sigma\left(1-(-\log G(z))^{-\xi}\right)\right]
$$


The number $G(z) \in[0,1]$ can be generated by the uniform (pseudo) random number generator, see for further details: Bratley, Fox \& Schrage (1987), Banks, Carson, Nelson \& Nicol (2001).

\section{STEP 2}

Secondly, using Monte Carlo simulations, we obtain 50,000 different paths for the Euribor rate $R_{t}, t \in[0,5]$. To implement geometric Brownian motion and model its movements, we use, in our simulation, the methodology based on Iterative Stochastic Equations (ISE). This method uses the concept of local characterizations for the Levy processes. A short description of this approach is provided below while for further details see Romaniuk M. (2003).

Let $[0, T]$ denotes the life time interval for the CAT bond. We discretize $[0, T]$ into a set of time moments $\tau=\left\{t_{0}=0, t_{1}, \ldots, t_{n}=T\right\}$, where $n$ is the number of steps. We assume that distances between points in the set $\tau$ are constant, in our simulation we take the step equal to one day, $\Delta t=t_{i+1}-t_{i}=$ const for $i$ $=1,2, \ldots, n-1$.

Now after the discretization, equation (2.3.3) may be replaced by the following iterative scheme

$$
R_{t+1}=R_{t} \exp \left(\left(\mu_{2}-\frac{1}{2} \sigma_{2}^{2}\right) \Delta t+\sigma_{2} \sqrt{\Delta t} \varepsilon_{i}\right)
$$

where $\varepsilon_{0}, \varepsilon_{1}, \ldots, \varepsilon_{n}$ are independent, identically distributed random variables from $N(0,1)$ distribution. This sequential form (4.2.3) is called an Euler scheme. The numbers $\varepsilon_{0}, \varepsilon_{1}, \ldots, \varepsilon_{n}$ can be generated by the normal (pseudo) random number generator, see also: Bratley, Fox \& Schrage (1987), Banks, Carson, Nelson \& Nicol (2001). The Statistical Toolbox of MatLab simplified our target, as there is a generator for random numbers of the typical Normal distribution. Therefore, the statistical analysis of the Euribor historical data from 1999 until June 2005 gave us the following estimates for the parameters: $\mu_{2}=0.032$ or $3.2 \%$ and $\sigma_{2}=0.01$. For the initial value $R_{0}$ of the equation (4.2.3), we assume $R_{o}=0.02082$ or $2.082 \%$ which refers to the actual value in June 2005 for the Euribor rate.

\section{STEP 3}

The third important step is the generation of sequences for the annual rate of a typical bank deposit account. Recall that, we have assumed that $i_{n}$ is a lognormally distributed random variable with parameters $\mu_{1}$ and $\sigma_{1}^{2}$ for any $n=$ $1,2,3, \ldots$, i.e. $i_{n}: \operatorname{LogNormal}(-3.94,0.061)$. The sequences are also obtained using the respective generator found in the Statistical Toolbox of MatLab.

\section{STEP 4}

The fourth step is the calculation of the payments (cash-flow stream) of the CAT bond for the five year period for all the 50,000 simulation. It can be easily concluded that this procedure is quite complex and requires logical functions and many subroutines. It should be mentioned that a catastrophic event may diminish 
our capital if and only if the maximum magnitude level of the sequence of 5 earthquakes is above 6.6. Looking carefully in Table 2 , the possibility of losing capital is below 3\%. That means, we have a 97\% Capital guarantee CAT bond which makes it quite attractive for conservative investors. Moreover, we assume a value for the face amount $€ K=1,000$ and a certain value risk premium $e=0.050$ or $5 \%$. While, the risk free rate continuously compounding (up to maturity date) is $r=0.027$ or $2.7 \%$ as for the five year Greek government bond (Bank of Greece, June 2005).

\section{STEP 5}

The last step is the calculation of future values at time $T=5$ according to equations (2.3.5) and (2.3.6) and then averaging over all the discounted values, we obtain the price of the CAT bond approximately equal to $€ 786.5$.

\section{CONCLUSION}

In this paper, we have tried to provide a short but comprehensive technical review for the design and implementation of risk-linked securities and especially the CAT bonds. That may be very useful for insurance practitioners and experts from the finance industry and the governmental authorities worldwide who realize that the CAT bonds demonstrate an innovative solution for improving the diversification of several different insurance risks.

Moreover, we have developed a practical example for covering earthquake risk in the boarder area of Greece estimating the respective probabilities using the tools of Extreme Value Theory. The proposed security has a non-indemnity-linked structure. It cancels the annual coupon payments or/and reduces the capital repayment at the maturity date whenever the triggering variable (i.e. the magnitude of the earthquake) raises beyond certain levels of the local magnitude scale.

Having estimated a Weibull type distribution for the risk dynamics of the magnitude of the earthquake and adapting, (for easiness of use) the Geometric Brownian motion for the risk dynamics of the Euribor rate (which determines the respective coupon payments), a certain price for the catastrophe bond is obtained. It is profound that the CAT bonds' price is more attracted to single investors, because of the higher yields. The investor of such bonds should not overlook the duration which is larger than the duration of similar straight bonds, as a consequence of the existence of the extra premium rate for bearing earthquake risk. Thus, the CAT bonds are more sensitive to movements in interest rates. Additionally, an intensive positive correlation between the CAT bonds' rate and the financial market returns is enhanced by the existence of this extra premium risk rate.

Further research is directed to the more complex problem of pricing an indemnity-linked security. Under that structure, coupon or capital payments are related with the level of the actual insured losses. The modeling of this problem should be developed in three stages by estimating: 
a) The distribution for the level of the magnitude of the earthquake.

b) A multivariate distribution for the basic characteristics of the respective earthquakes e.g. the location, depth, speed etc.

c) The distribution for the potential damages and losses after a certain type of earthquake.

\section{ACKNOWLEDGMENTS}

The authors wish to thank the anonymous referees for their comments which improved the final quality of the manuscript.

\section{REFERENCES}

Bank of Greece (2005) Financial Bulletin (in Greek) 25, August 2005.

Banks, J., Carson, S.J. II, Nelson, L.B. and Nicol, M.D. (2001) Discrete-Event System Simulation. Prentice Hall International Series in Industrial and System Engineering, U.S.A.

Baryshnikov, Y., Mayo, A. and TAYlor, D.R. (2001) Pricing of CAT Bonds.

Baxter, M. and Rennie, A. (1999) Financial Calculus: An introduction to derivative pricing. Cambridge University Press, U.K.

Beelders, O. and Colarossi, D. (2004) Modeling Mortality Risk with Extreme Value Theory: The Case of Swiss Re's Mortality-Indexed Bonds. Global Association of Risk Professionals 19, 26-30.

Beirlant, J., Teugels, J. and Vynckier, P. (1996) Practical Analysis of Extreme Value. Leuven University Press, Belgium.

Boyle, P., Broadie, M. and Glasserman (1997) Monte Carlo methods and security pricing. Journal of Economics 21, 8-9

Bratley, P., Fox, L.B. and Schrage, E.L. (1987) A Guide to Simulation. Springer, $2^{\text {nd }}$ Edition, U.S.A.

BRIYS, E. (1997) From Genoa to Kobe: Natural Hazards, Insurance Risks and the Pricing of Insurance-Linked Bonds. Lehman Brothers International, London U.K.

Coles, S. (2004) An Introduction to Statistical Modeling of Extreme Values. Springer Series in Statistics, $3^{\text {rd }}$ edition, Great Britain.

Coles, S. (2004) "S-plus Functions for Extreme Value Modeling". http://www.maths.bris.ac.uk/ masgc/ismev/summary.html.

Cox, H.S. and Pedersen, W.H. (2000) Catastrophe Risk Bonds. N.A.A.J. 4(4), 56-82.

Cummins, D. and Geman, H. (1995) Pricing Catastrophe Futures and Call Spreads: An Arbitrage Approach. Journal of Fixed Income 4, 46-57.

D'Archy, S.P. and France, G.V. (1992) Catastrophe Futures: A Better Hedge for Insurers. Journal of Risk and Insurance 59, 575-600.

Embrechts, P., KlüPpelberg, C. and Miкosch, T. (2003) Modelling Extremal Events. Springer, $4^{\text {th }}$ edition, Germany.

Embrechts, P., Resnick, I.S. and SAmorodnitsky, G. (1999) Extreme Value Theory as a Risk Management Tool. N.A.A.J. 3(2), 30-41.

EURIBOR Historical Data (EURIBOR organization): http://www.euribor.org

FisheR, R.A. and TIPPETT, L.H.C. (1928) On the estimation of the frequency distributions of the largest or smallest number of a sample. Proceedings of the Cambridge Philosophical Society 24, 180-190.

GNEDENKo, B.V. (1943) Sur la distribution limite du terme maximum d'une série alétoire. Annals of Mathematics 44, 423-453.

Hosking, J.R.M. (1986) Maximum-likelihood estimation of the parameters of the generalized extreme-value distribution. Applied Statistics 34, 301-310.

Institute of GeODYNAMICS, National Observatory of Athens (IG-NOA). At World Wide Web address: http://www.gein.noa.gr/services/cat.html

Jenkinson, A.F. (1969) Statistic of Extremes. Technical Note 98, World Meteorological Organization. Chapter 5, 183-227.

Kellison, G.S. (1991) The Theory of Interest. Irwin/McGraw-Hill. U.S.A. 
Kotz, S. and Nadarajah (2002) Extreme Value Distributions: Theory and Applications. Imperial College Press, Singapore.

LeE, J.-P. and Yu, M.-T. (2002) Pricing Default-Risky CAT Bonds with Moral Hazard and Basis Risk. The Journal of Risk and Insurance 69(1), 25-44.

Loubergé, H., Kellezi, E. and Gilli, M. (1999) Using Catastrophe-Linked Securities to Diversify Insurance Risk: A Financial Analysis of Cat Bonds. Journal of Insurance Issues 22, 125-146.

Macleod, A.J. (1989) Comment on Maximum-Likelihood Estimation of the Parameters of the Generalized Extreme-Value Distribution. Applied Statistics 38, 198-199.

MCNeIL, J.A. (1997) Estimating the tails of Loss Severity distributions using Extreme Value Theory. ASTIN Bulletin 27(1), 117-137.

MCNEIL, J.A. (2001) "EVIS, version 4". http://www.math.ethz.ch/ moneil/software.html

Miller, G. (Senior Vice President and Deputy General Counsel of the Bond Market Association) and De Konkoly, Th.M. (Vice President and Associate General Counsel of the Bond Market Association) (2002) Comments on Draft GAO report "Catastrophe Insurance Risks: The Role of Risk-Linked Securities and Factors Affecting their Use". GAO-02-941

NUTTER, W.F. (President of Reinsurance Association of America (RAA)) (2002) Comments on the GAO's preliminary report entitled "Catastrophe Insurance Risks". GAO-02-941.

O'Brien, T. (1997) Hedging Strategies Using Catastrophe Insurance Options. Insurance: Mathematics and Economics 21, 153-162.

ØKSENDAL, B. (2003) Stochastic Differential Equations. Springer, $6^{\text {th }}$ edition, Germany.

Papanastassiou, D., Latoussakis, J. and Stavrakakis, G. (2001) "Proceedings of the 9th International Congress of the Geological Society of Greece", Athens, September 2001. Bulletin of the Geological Society of Greece XXXIV/4, 1563-1566.

Prescott, P. and Walden, A.T. (1980) Maximum Likelihood Estimation of the Parameters of the Generalized Extreme-Value Distribution. Biometrika 67, 723-724.

Prescott, P. and WAlden, A.T. (1983) Maximum Likelihood Estimation of the Parameters of the Three-Parameter Generalized Extreme-Value Distribution from Censored Samples. J. Statist. Comput. Simulation 16, 241-250.

RESNICK, I.S. (1997) Discussion of the Danish Data on Large Fire Insurance Losses. ASTIN Bulletin 27(1), 139-151.

Romaniuk, M. (2002) Pricing the risk-transfer financial instruments via Monte Carlo methods. Interium Report IR-02-065 for International Institute for Applied Systems Analysis (Approved by Ermoliev Yuri).

RomAniuk, M. (2003) Pricing the risk-transfer financial instruments via Monte Carlo methods. System Analysis Modelling Simulation 43(8), 1043-1064.

United States General Accounting Office (2002) Catastrophe Insurance Risks: The Role of Risk-Linked Securities and Factors Affecting their Use. GAO-02-941.

United States General Accounting Office (2005) Catastrophe Risk: U.S. and European Approaches to Insure Natural Catastrophe and Terrorism Risks. GAO-05-199.

United States General Accounting OfFice (2006) Federal Emergency Management Agency: Challenges for the National Flood Insurance Program. GAO-06-335T.

Vaugirard, V. (2003) Pricing Catastrophe Bonds by an Arbitrage Approach. The Quarterly Review of Economics and Finance 43, 119-132.

Vaugirard, V. (2004) A Canonical First Passage Time Model to Pricing Nature-Linked Bonds. Economic Bulletin 7(2), 1-7.

Young, R.V. (2004) Pricing an Incomplete Market with in an Affine Term Structure. Mathematical Finance 14(3), 359-381.

Alexandros A. Zimbidis

Nickolaos E. Frangos

Athanasios A. Pantelous

Department of Statistics

Athens University of Economics and Business

Patision 76, 10434 Athens, Greece

E-mails:aaz@aueb.gr,nef@aueb.gr,apantel@aueb.gr 